\title{
Multiple Input Multiple Output (MIMO) Model of an Industrial Ecosystem: An Agent-Based Modeling Approach
}

\author{
Ganiyu O. Ajisegiri \\ Department of Industrial and Production Engineering \\ Faculty of Technology \\ University of Ibadan \\ Nigeria \\ go.ajisegiri@ui.edu.ng
}

\author{
Frans L. Muller \\ School of Chemical and Process Engineering \\ Faculty of Engineering \\ University of Leeds \\ Leeds, United Kingdom \\ f.l.muller@leeds.ac.uk
}

\begin{abstract}
This paper addresses the application of the AgentBased Model (ABM) to simulate the evolution of Multiple Input Multiple Output (MIMO) eco-industrial parks to gain insight into their behavior. ABM technique has proven to be an effective tool that can be used to express the evolution of eco-industrial parks. The ABM represents autonomous entities, each with dynamic behavior. The agents within the eco-industrial park are factories, market buyers, and market sellers. The results showed that the Réseau agent-based model allowed the investigation of the behaviors exhibited by different agents in exchange for materials in the industrial park.
\end{abstract}

Keywords-eco-industrial park; agent-based model; Réseau; supply; demand

\section{INTRODUCTION}

In the last two decades, attention for Eco-Industrial Park (EIP) development projects has grown enormously among national and regional governments and industries [1]. National Industrial Symbiosis Program (NISP) in the UK is an example of numerous industrial ecosystems [2,4]. It is believed that a well-planned, functioning EIP has the potential to benefit the economy and to substantially relieve environmental pressure in and near the location of its development [1, 5-7]. It is intuitive that the utilization of a previously unused (e.g. in the case of released steam) or discarded (e.g. sludge to landfill) resources can typically offer financial savings to both the utilizing company and the provider while reducing the use of raw materials, energy or water, and avoiding disposal to landfill. The benefits of Industrial Symbiosis (IS) include but are not limited to social benefits (may include job creation), emission reduction (e.g. gaseous pollution or dust), and aesthetic improvements (e.g. reduction of waste piles).

The aim of the present work is to apply an agent-based model to simulate the evolution of a Multiple Input Multiple Output (MIMO) EIP. Agents are described in detailed standard protocol [8] for describing individual-based and agent-based models. Due to the short fall in the initially developed model, which only accommodates Single Input Single Output (SISO) agents the model is modified to allow many MIMO agents (in the range of thousands). The simulation of an MIMO EIP system using ABM is the novelty of this work.

\section{RELATED WORK}

Since the emergence of industrial ecology in the 1950s and its take-off during the 1990s, much progress, in theory, policy, and practice has been achieved for designing fruitful and sustainable EIPs. Almost all research into IS/EIP systems involves either proposing a frame work [9] or a mathematical model [10-11] for the IS/EIP design. System modeling and computer-based simulation techniques have been applied to different problem areas [12] and are more flexible in predicting complex phenomena due to their dynamic behavior. There are a few works [13-15] that focus on the simulation of IS to understand its complexity. Authors in [16] proposed a theoretical model for the emergence of eco-industrial parks at different levels, but they did not implement it into a simulation model. There is still progress to be made in the area of computational modeling of the actions and interactions of the autonomous agents that form an EIP. Major problems in unraveling the complexity of IS-based on demand response [17] include but are not limited to price, profit, and supplydemand fluctuations. Also, part of the problem that exist in the design of EIP systems is the mismatch between the supplying and demanding agents at any time resulting in the occurrence of periods of excess supply (supply greater than demand) and shortage (demand exceeds supply). This paper intends to fill these gaps. Agent-based models have been used when there is the need to model the dynamics of circular economies and IS networks [17-18]. In an agent-based model, actors (or agents) interact using prescribed rules, and the emergent behavior of the system is observed [18-20]. ABM, also known as bottomup modeling, [21], has proven to be a promising tool to simulate the evolution of EIPs [15, 22].

After an up-to-date literature review and to the best of our knowledge, this is the first attempt of simulating MIMO industrial ecosystems to forecast supply and demand time series using ABM integrated with an input-output model. We focus on the application of an agent-based model to the design 
of EIP in which price fluctuation and demand variability are emergent properties of the interaction among the agents. Réseau agent-based model [23], is used to simulate the industrial ecosystem and generate demand and supply profiles. We analyze IS considering materials and energy flows and the related supply-demand match for each output products (finished goods, by-products, useful waste) becoming primary input for entirely new processes that are co-located or within the same vicinity. We simulate the effect of price, demand and supply fluctuations to express the dynamics of IS/EIP systems.

\section{ABM BASICS}

ABM is a class of computational modeling methods that simulate individuals making decisions according to programmable rules. Those rules are set by the modeler to represent key elements of real world decisions, including the individuals' own characteristics and their social and physical environment. An agent, in a logistics context, might represent a machine, the order handling process, inventory handling and further production planning and scheduling. The definition of an intelligent agent suggests the following properties to be inherent:

- Autonomous: functions without the need for user intervention.

- Proactive: operates independently towards a goal.

- Reactive: responds to changes in the environment and changes in the course of action.

- Social: interacts with other agents in order to exchange required information.

\section{A. Why ABM for Modeling Industrial Ecosystems?}

In the last 5 years, the majority of the research works focused on the use of input-output models and ABMs separately to promote IS. However, this is not sufficient to reveal the complexity of the concept. Authors in [24] designed IS using input-output approach. Even though their work introduces the concept of perfect symbiosis to enhance the future production area, it lacks in promoting the drivers (e.g. price, demand and supply) that enhance cooperation decision of the participating factories. Since input-output model and ABM are promising tools, we explore the possibility of combining these two methods together for the modeling of the ecosystem. Majorly, the input-output model is embedded in the production chain of the factories in the IS/IES while ABM is used for the entire IS components.

\section{B. Validation of $A B M S$}

One of the main valuable aspects of a simulation model is its validity. Validation is the process of determining whether the programming implementation of the conceptual model is correct. Simulation validation is often considered using Zeigler's hierarchy of model validity $[8,25]$, that is, replicative, predictive, and structural. There are different variations on this calcification [26, 27], but for the purposes of ABM validation, Klügl used the validity along two dimensions [28] using only two levels: (1) Face validation and (2) Empirical validation. Face validity shows that the simulation processes and the output conform to human judgment within the frame of theoretic basis and implicit knowledge of experts while validation through empirical means is done by using statistical measures and tests to compare key figures produced by model with numbers gathered from the reference system.

\section{MODEL SUMMARY AND IMPLEMENTATION}

$\mathrm{ABM}$ is extensively used within the complexity theory and springs from object-oriented programming and distributed artificial intelligence. The agent formulations used in this work extend the basic formulation of [29]. The Overview, Design concepts and Details (ODD) protocol proposed by $[30,31]$ is used for the model's description. It was designed in a way that ABM publications would be more complete, quick and easy to understand, and organized in a manner that allows presenting information in a consistent order [30]. The model is an integrated agent-based model with input-output approach and was developed with Python, which is a general-purpose programming language. Following the ODD protocol for describing individual-based and agent-based models, this section describes the elements of ODD as related to the proposed Réseau-EIP ABM.

\section{A. Overview}

The overview of the ODD consists of three elements, the model purpose, state variables and scales, and process overview and scheduling. These are explained below.

\section{B. Model Purpose}

The Réseau-EIP ABM is constructed as a decision making tool for understanding the emergence behavior that favours the design of EIPs. The model is intended to be used in assessing the sustainability of an EIP and by improving its economic, environmental, and social performance. The model is used to simulate the effects of price and supply and demand fluctuations to express the dynamics of the EIP system. In the future, the model will be improved to estimate the impact of the energy storage system in the design of the EIP, will incorporate "what-if" scenarios", generate hypotheses and test policy ideas related to the EIP development policy.

\section{Entities, State Variables and Scales}

The model consists of two core entities called market and factory agents. The factory agents represent industrial plants within a network and links, which represent the exchange of resources, while the market agents on the other hand do not produce anything but only buy finished goods or sell raw materials. As indicated earlier, EIP involves sustainable exchange of resources among partners within the park. Therefore, a raw material to a plant can be an output of another plant. The factory agents are characterized by state variables: factory agent identification number (ID), raw materials type, raw materials stock, raw materials usage, products (finished, by-product and waste) name, product price, price variance, output capacity of a product type (product target), net worth and the location ( $\mathrm{x}$ and $\mathrm{y}$ co-ordinates) of the factory agent within the EIP network. There are two market agents: the selling agent and the buying agent. The selling agent is characterized by the state variables: selling agent ID, product (goods) name, selling price, price variance product type 
(product target), net worth balance, and location. The buying agent is characterized by: buying agent ID, raw material type, demand quantity, net worth, and location. In the model, all the agents (factory, selling and buying) interact with each other. The factory agent at a time step fulfills its input requirement (based on product demand) by initiating a contract with a selling agent. After getting the input materials, the factory agents begin to produce, determine product prices, and sell to the buying agents. Since the factory agents buy raw materials and sell the output, they can compete with the market agents. A monthly time step is chosen for this work but any time step (daily, weekly etc.) can be chosen. The model is grid-based and there is no specific dimension used. Each agent has its $\mathrm{x}$ and $\mathrm{y}$ coordinates to indicate its location on the grid. The grid served as the Réseau-EIP boundary. No interface or visualization is built in with the model and the output of the simulation is exported to an excel file and necessary analysis is performed thereafter.

\section{Process Overview and Scheduling}

The Réseau-EIP ABM runs with monthly time steps. Within each month or time step, six different submodels run in succession. Each of these submodels is discussed briefly here and a full discussion of each submodel can be seen in the Detail section. At the beginning of the simulation and for each time step, the factory, buyer, and seller modules load their variables and parameters from an external file, predict production (factory agent) and determine prices. While the interaction of the three agents from these modules evolves in a time step, the transaction module begins and handles the contract between buyers and sellers. The history module runs next and records the history of each of the agents. The reporting module runs last and reports all the outputs of all the agents in an external file.

\section{E. Design Concepts}

\section{1) Interactions}

Factory agents interact with each other and with the market agents (they buy input materials, and they sell output goods). The primary interaction between agents is the exchange of resources. In the buying and selling submodels, a buyer (factory or market buying agents) establishes contract with sellers (factory or market selling agent) through the transaction submodel, in which, based on the quantity of available goods and the price, the buyer enters a contract with the sellers and purchases its raw materials from the best seller (cheapest price). The agents also interact by imitating each other's attributes.

\section{2) Sensing}

All agents are assumed to know their own attributes. It is also assumed that agents are also aware of their environment. This information agent informs factory, buying, and selling agents to make decisions at any time.

\section{3) Emergence}

The dynamics of the park and resource exchange demonstrate emergence based on the lower level interactions and decisions of the factory and the market (buying and selling). Therefore, the important thing from the model is the emergence of the net worth value of each agent based on the agent's interaction with the other agents.

\section{4) Adaptation}

Adaptation is modeled explicitly in the Réseau-EIP ABM model. Agents adapt to supply and demand requests by finding new partners to exchange goods with. Each factory agent always looks for raw materials to purchase either from another factory's agents or from market selling agents in order to produce its output and sell it to waiting buyers (factory or market buying agents).

\section{5) Learning}

Each agent in the core entities of this model learns from its history by using a learning procedure to make decisions at every time step. An example is the history of the prices of goods in the market. The agents always check the previous price and based on the Weibull distribution function make a decision either to change (increase or reduce) or maintain the price for the next time step.

\section{6) Prediction}

At present, the agents in the Réseau-EIP ABM model do not use any prediction models to make decisions.

\section{7) Stochasticity}

Stochasticity plays a vital role in the Réseau-EIP ABM model. At the beginning, each agent loads its parameters from an input file and some random distributions add an element of stochasticity into all subsequent runs.

\section{8) Objectives}

All agents in the model do not only seek to collectively maximize their "purpose", but instead make decisions to buy, sell, produce goods, and determine price as autonomous entities. At each decision period, agents make decisions in accordance with the sensed data and with a set of random techniques.

\section{F. Details}

\section{1) Initialization}

The Réseau-EIP ABM model is initialized by using data obtained from the relevant literature. There are three different agents (factory, buying, and selling). The variables with their parameters for each agent are organized in an external excel file and the agents pre-load their data. Based on this, users can therefore run different scenarios by varying the input parameters and observing their impact on the output.

\section{2) Input Data}

The input data are Excel-ased and are specific for each agent. Apart from the initialization data, no other data are required to run the model. The input file is user friendly and users can easily change the parameters to suit the problem in question.

\section{3) Submodels}

\section{a) Parameter Loading}

Each agent loads its parameters from an input file. The parameters are agent specific with a unique identifier. 


\section{b) Requirement Prediction}

This method is used by all the agents to predict their requirements at every time step. This method is based on the Gaussian distribution. The market agent demand is equivalent to the requirement predicted while the factory agent has two variables to determine at the beginning of each time step. These are the sales quantity and price. These two variables are also modeled using the Gaussian distribution. The market selling agents only predict the selling price of all its goods.

\section{c) Production Step}

Since the buying and selling agents do not produce anything, they are only associated with selling agents with readily available goods to be sold. In contrary, the factory agents produce goods therefore a production method is included in the factory class as an input-output ratio formulation.

\section{d) Purchasing Method}

Only the buying agents (factory and market agents) buy goods/raw materials. Therefore, this method is only associated with these two agents. For each time step, each buyer checks all the prospective sellers and makes contact with all the sellers that have the sufficient quantity of goods. The sellers are appended in a list and the buyers buy from the cheapest seller until their requirements are fulfilled.

\section{CASE STUDY}

Figure 1 shows the potential structure of the studied MIMO industrial ecosystem. It includes three different kinds of factories: (1) bio-refinery plants (BIOs), (2) combined heat and power plants (CHPs), and 3 ) anaerobic digestion plants (ADs). The AD plants convert organic waste to biogas and generate some residual waste. They use process steam and electricity to heat up the digester. CHP plants can use biogas as a replacement for coal, while generating electricity and process steam. It should be noted that fuel switching (i.e. biogas as a replacement for coal) cannot be undertaken easily and is beyond the scope of this work. The BIOs' main output is ethanol while they generate lignin pellet and waste water as byproducts. These three candidate plants form the internal environment of the industrial ecosystem while the market sellers and buyers come from the external environment of the park where raw materials can be purchased or finished goods can be absorbed without any capacity limitation. The internal environment consists of three different stages. Each stage is made up of three different firms that produce the same output but use different input raw materials. Stage A includes three different $\mathrm{AD}$ plants that produce biogas as their main product. To produce biogas, the plants require either cattle feedlot manure or food and bio-solids [11, 32]. Stage B consists of three different CHP plants that produce electricity and process steam (heat). Lastly, stage $\mathrm{C}$ is made up of BIO plants that generate ethanol as their main output. Each firm and market seller exhibit a stochastic demand over time, distributed according to the Gaussian distribution with a given mean and standard deviation. The Réseau is used to simulate the interaction between companies, market buyers and sellers in the IES in order to understand the dynamic behavior of the participants in the park and particularly the process plants. The initial data for the $\mathrm{AD}$ and CHP plants were obtained from [11]. The three CHP plants separately have a capacity for biogas (methane) ranging from $80,000-500,000 \mathrm{~m}^{3}$ per month while the $\mathrm{AD}$ plants utilize food and bio-solid wastes in the range of 0.3 million tons and required energy within $30-$ $65 \mathrm{MWh}$.

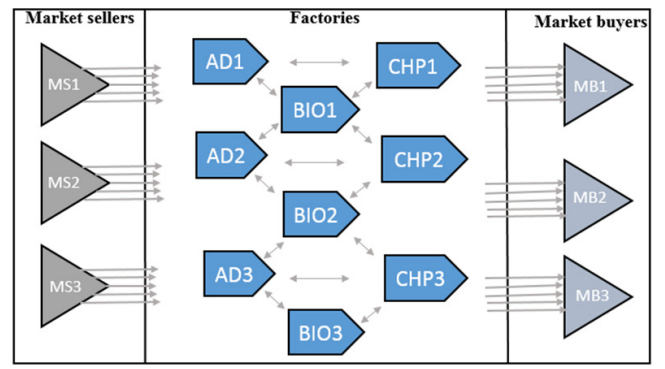

Fig. 1 Configuration of the MIMO industrial ecosystem.

\section{SIMULATION RESULTS AND DISCUSSION}

This section focuses on the results obtained by the simulations of the IES with MIMO factory agents shown in Figure 1. The simulation output of the case study demonstrates the effectiveness of the proposed method. The simulation was carried out in two different ways. Firstly, the simulation was done to determine the behavior and the way each factory makes decisions operating in standalone mode, in which each factory only buys from the market or sells to the market agents while the other factory agents are absent. The second simulation was carried out with the presence of all factory agents in the IES. Thereby possible symbiotic relationships can occur between the agents and/or the market agents.

\section{A. Demand and Supply Response}

The results of a single simulation run for the generation of demand/supply for the factory agents in the industrial ecosystem are shown in Figures 2 and 3. Each of the Figures, is divided into four subfigures. The division shows the different demand/supply curves for the different decision strategy types used during the simulation. Note that one simulation cycle stands for a time period of one month. As stated above, two different decision rules from the buyer and seller agents are used. For the buyer agent, random and risk based decision strategies were considered while random and best price were used as the decision making strategies for the sellers. The combination of the decisions gives different simulation results. As seen in Table I, there are four (I - IV) decision Strategy Types (STs) that are used to assess the behavior of the agents in the IES.

TABLE I. BUYER/SELLER DECISION STRATEGY TYPES

\begin{tabular}{|c|c|c|c|}
\hline \multicolumn{2}{|c|}{} & \multicolumn{2}{|c|}{ Seller } \\
\cline { 3 - 4 } \multirow{2}{*}{ Buyer } & BDS1 & SDS1 & SDS2 \\
\cline { 2 - 4 } & BDS2 & ST I & ST II \\
\hline
\end{tabular}

For example, using ST I, the buyer enters into the market, purchases randomly (random price of materials) while the sellers change their prices randomly according to the market 
price history. The figure can explain the synergy that occurs in the ecosystem when the agents freely interact with each other to exchange resources.

(a)

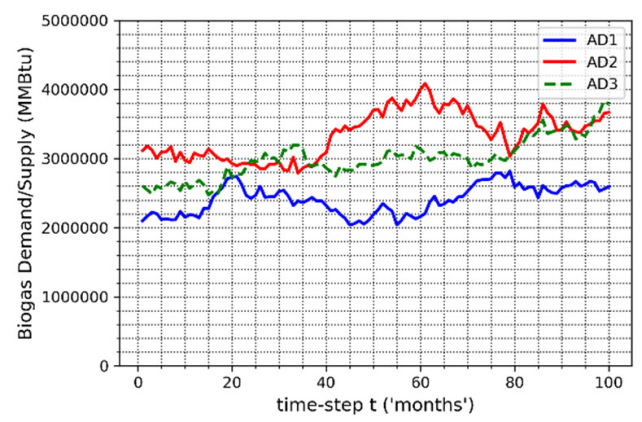

(b)

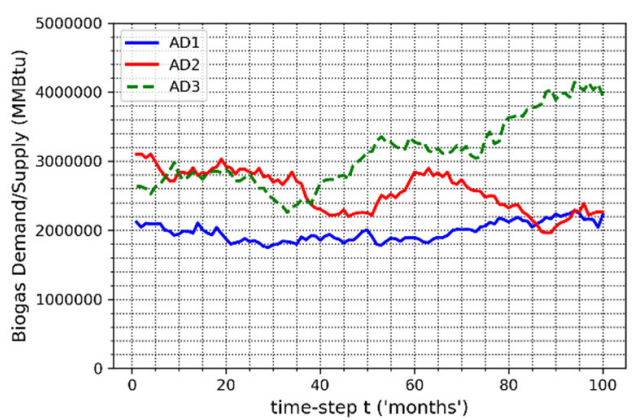

(c)

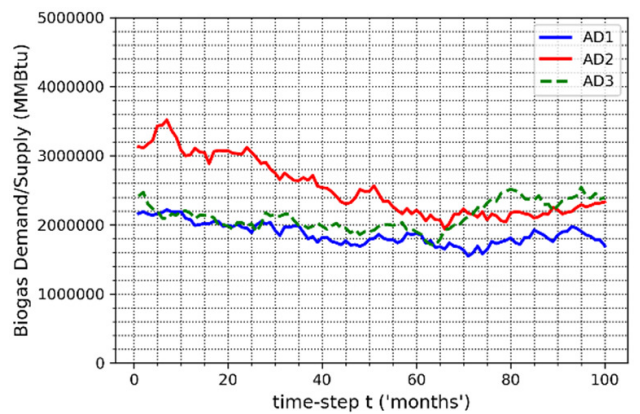

(d)

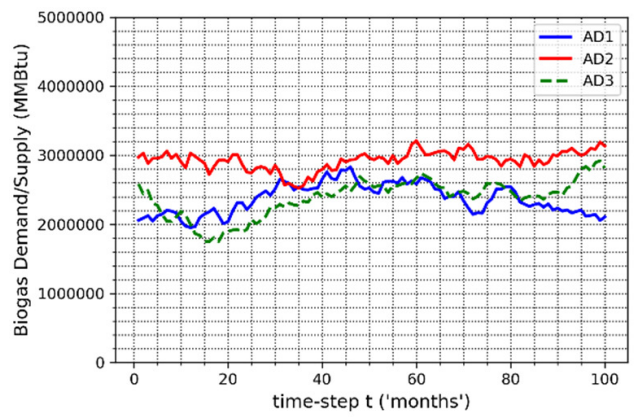

Fig. 2 Average biogas demand/supply time series: (a) ST I, (b) ST II, (c) ST III, (d) ST IV.

Figure 6 shows the symbiotic relationship that exists between the three combined heat and power plants as buyers (CHP1, CHP2, CHP3) and the anaerobic digesters as sellers (AD1, AD2 and AD3). Figure 2 shows the biogas consumption (volume) of the three combined heat and power plants (agents) in the IES. It can be seen that the average demand and supply for the three CHP plants is $2.6 \times 106 \mathrm{MMBtu}$ over the period. When the simulation starts, the biogas usage for the three CHP plants is approximately the same for the four different decision strategy types by the buyer-seller agents.

(a)
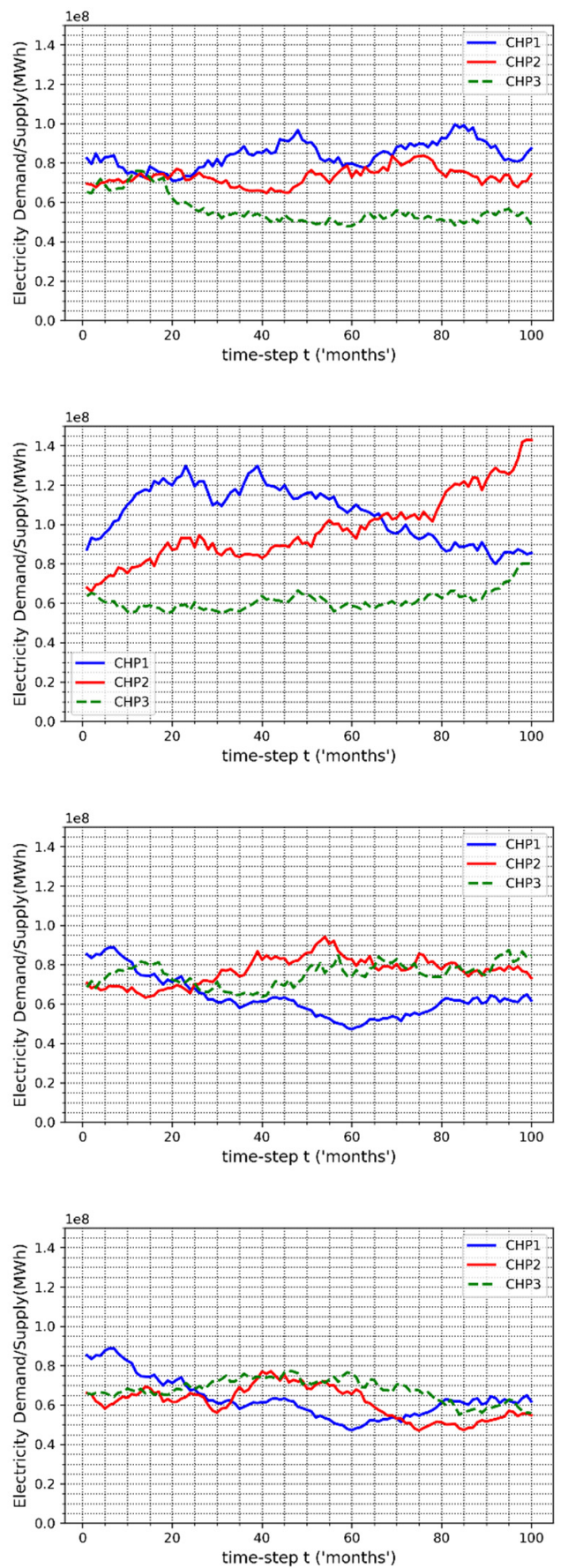

Fig. 3 Average electricity demand/supply time series: (a) ST I, (b) ST II, (c) ST III, (d) ST IV. 
This happens because the initial input parameters are the same at the beginning of the simulation runs. After some simulation cycles, it can be seen that biogas usage reaches its maximum point and afterwards it declines. The fluctuation in the total usage of biogas is linked to the saturation in the biogas usage in the IES and it is also being affected by the decision strategy type the agents are using during the market transaction. In comparison to the contribution of the selling agents in the IES, agent AD2 supplies are the highest over the period except in Figure 2Error! Reference source not found.(b) where AD3 supplies more from the 40th simulation cycle onward.

The average supply of biogas in the IES is $2.25 \times 106$, $2.3 \times 106$, and $2.5 \times 106 \mathrm{MMBtu}$ over the period for agents AD1, $\mathrm{AD} 3$, and $\mathrm{AD} 2$ respectively. The assumptions made under the BIO plant configuration, suggest that BIO plants have the potential to synergize with both the $\mathrm{AD}$ and $\mathrm{CHP}$ plant agents. The demand/supply curves between the three BIO plants as seller agents (BIO1, BIO2, $\mathrm{BIO} 3)$ and the $\mathrm{AD}$ plants (AD1, $\mathrm{AD} 2$ and $\mathrm{AD} 3$ ) and $\mathrm{CHP}$ plants (CHP1, CHP2, CHP3) as the buying agents were also simulated. The results show the Distilled Dry Grain (DDG) sold out per month by BIO agents to the AD agents. Agent BIO2 supplied the least DDG for the four different decision making rules. This is a result of the agent $\mathrm{BIO} 2$ having the lowest production capacity compared to the other two BIO agents. Apart from this, the input/output ratio for $\mathrm{BIO} 2$ is also comparably low. On average, about $2.7 \times 108 \mathrm{lb}$ of DDG is sold in the market over the entire period. The BIO2 sales of DDG are stable throughout the simulation period. For the other two BIO agents, the supply of DDG fluctuates but the peaks are considerably lower at any given interval. BIO agents have a strong symbiotic link with $\mathrm{CHP}$ plants. The lignin pellet average demand/supply for the three CHP plants is $0.5 \times 106$ tons over a period. The fluctuation in the total usage of lignin pellet is linked to saturation in its usage in the IES and it is also being affected by the decision strategy type the agents are using during the market transaction. The average supply of lignin pellet in the IES is $0.6 \times 106,0.53 \times 106$, $0.52 \times 106$ tons over a period for bio-refinery agents $\mathrm{BIO} 1$, $\mathrm{BIO} 2$, and $\mathrm{BIO} 3$ respectively. From the above, it can be concluded that there is a strong SRF in selling by-products between the BIO agents as sellers and CHP and AD agents as buyers in the IES. This is in line with the conclusions in [11]. The CHP plant agents have the potential to synergize with BIO, $\mathrm{AD}$ and market buyer agents. Figure 3 shows the average electricity sold to the BIO agents by the three CHP agents during the transaction. The impact of the CHP plants on the supply of electricity and process steam to the BIO plants is highly depended on the symbiotic index shown in Figure 6. Based on the synergy that occurs internally in the IES, it suggests that the BIO agent will transfer more with the external agents if the CHP plants are not part of the agents in the internal part of the IES. As shown in Figure 3, the supply is randomly distributed and the maximum supply is $0.85 \mathrm{MWh}$ by CHP1, except in Figure 3(d) where CHP2 supply reaches $0.73 \mathrm{MWh}$ while the minimum supply is about $0.6 \mathrm{MWh}$ in all the four figures. The average process steam supplied by agent CHP3 is the highest throughout the simulation period except when CHP2 supplied more to the BIO agents. However, in all the decision strategy types, agent CHP1 supplies the least and this is a result of the low output of the generated steam.
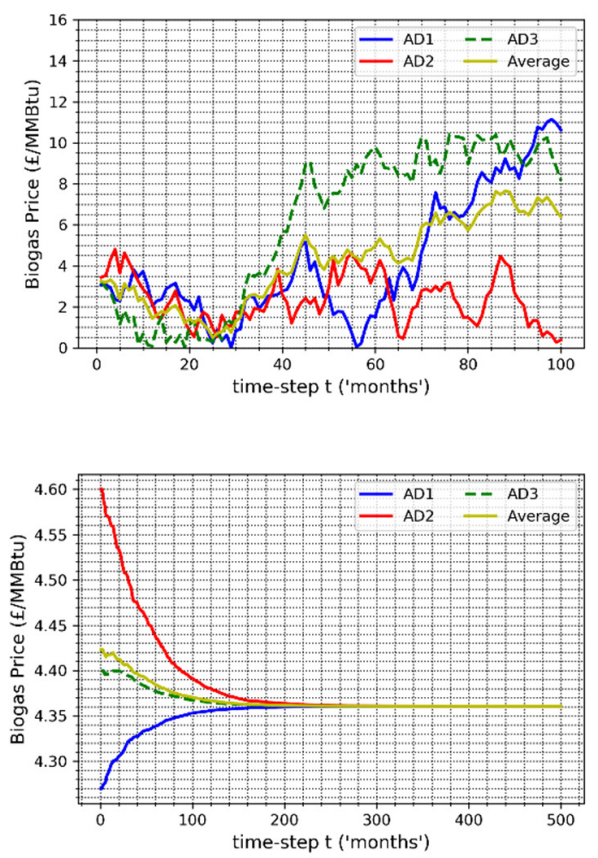

(c)

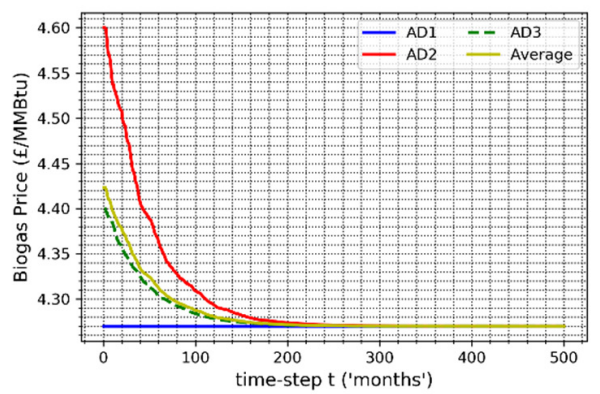

(d)

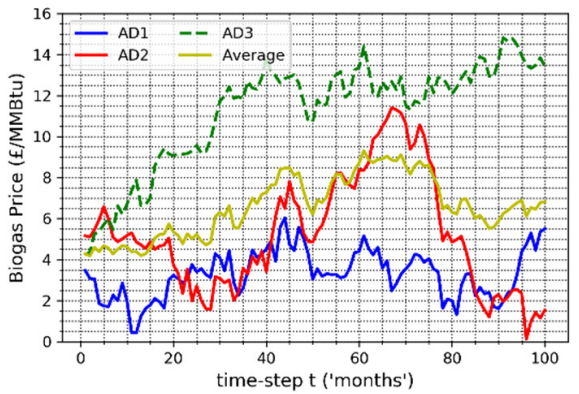

Fig. 4 Biogas price variation under different seller-buyer decision strategy types: (a) ST I, (b) ST II, (c) ST III, (d) ST IV.

\section{B. Price Evolution of Agents in the Industrial Ecosystem}

To showcase the evolution of prices over time, simulation results for two different organizations, i.e. biogas and CHPs, are presented here. Figures 4-5 present the price evolution of the material exchange in the IES for each of the factory agents. 
(a)

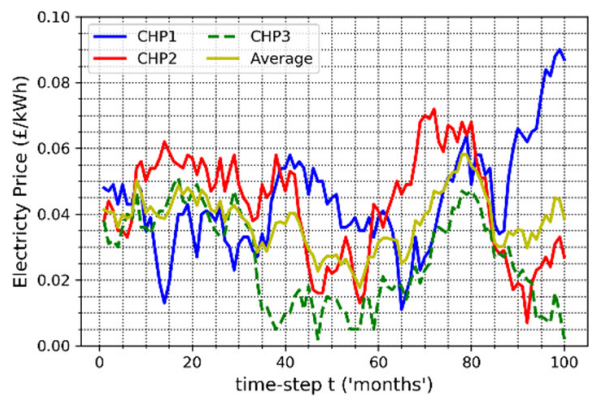

(b)

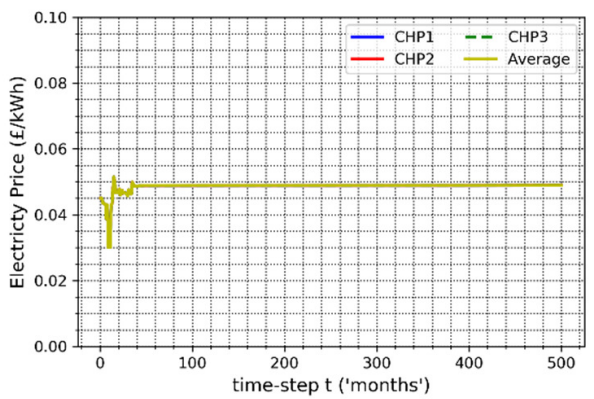

(c)

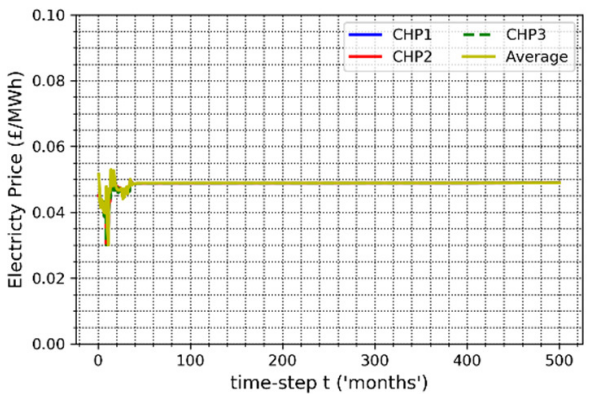

(d)

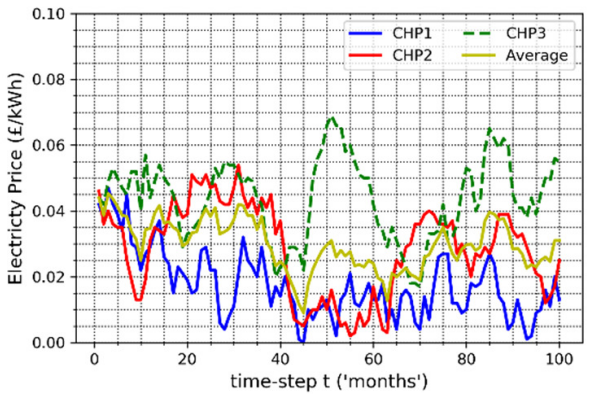

Fig. 5 Electricity price variation under different seller-buyer decision strategy types: (a) ST I, (b) ST II, (c) ST III, (d) ST IV.

Again, each Figure is divided into four subfigures, one for each different decision strategy type used in the simulation. As shown in Figures 4-5, we carried out 100 runs for ST I and ST IV while 500 runs were carried out for ST II and ST III. The reason for this difference was the need to show the price converge to a point in ST II and ST III after a time period. Sellers can change prices either randomly or after a strategy, while five different decision making strategies have been proposed for the buyers, although only two (random and best price) are used in this case study.

In Figures 4(a) and 4(d), the biogas price fluctuates and reaches $£ 14 / \mathrm{MMBtu}$. In reality, this cannot be used for decision making by business owners. Figure 4(b) and 4(c) on the other hand, can be linked to what happens in real life. At the beginning of the simulation runs the price changes and over time it moves towards equilibrium. The price of biogas flattens out at the $160^{\text {th }}$ period in both cases and remains the same until the end of the simulation run. This flattening happens as a result of the decision strategy used by the sellers (risk based) that forced the market price to move towards equilibrium when any change will mean fewer sales for the selling agent. However, there is little difference between the ST II and ST III. For the ST III, the equilibrium price shifts towards the agent with the lowest price in the IES, while there is a random shift at every period before equilibrium is reached in the ST II. The possible materials that the $\mathrm{BIO}$ can exchange are lignin pellet and DDG while process steam and electricity are the exchange materials for the CHP plants. Figure 5 shows the price variation for electricity and process steam under four different decision strategy types by the sellers and buyers. In this case, the CHP plants are the selling agents while the $\mathrm{BIO}$ and $\mathrm{AD}$ plants are the buying agents in the IES. The ST I and ST IV decision rules as pointed earlier are not realistic and this is indicated in the graph. The minimum average price of electricity as can be seen is approximately $£ 0 / \mathrm{MWh}$ and the maximum reaches as high as $£ 0.09 / \mathrm{MWh}$ in Figure 5(a) while the maximum value in Figure $5(\mathrm{~d})$ is $£ 0.08 / \mathrm{MWh}$. As can be seen, the price of all the selling agents did conform to the average price recorded from the market history.

From the simulation results, the sellers (BIO plants), act as offensive players while the buyers act as defensive players. When the decision strategy is either ST I or ST IV, the price of DDG fluctuates vividly. In the other two decision rules, the price reaches a peak as high as $£ 0.18 / \mathrm{lb}$ before converging and then the price $(£ 0.082 / \mathrm{lb})$ is maintained throughout the simulation. In case of the lignin pellet price variation, the average price fluctuates and reaches a maximum value of $£ 24 /$ ton, which is close to reality. For ST II and ST III, the price of lignin pellet is almost the same from after the $140^{\text {th }}$ period. The value in the case of ST II is $£ 6.2 /$ ton while it is $£ 6.16 /$ ton for the ST III.

\section{Buyer-Seller Symbiotic Relationship}

In order to identify changes of symbiosis in an IES, Figure 6 shows the Symbiotic Relationship Index (SRI) for each of the decision strategy types. As expected during the beginning of the simulation, the SRI value is close to 0.2 , which indicates the absence of symbiosis in the IES. This can be seen at the beginning of each of the simulation runs. While the simulations run, the SRI value grows significantly which indicates an increase in the transaction relationship between the different factories. It can be seen that the symbiotic relationship grows considerably from the 10th period and fourth, and this trend was maintained until the end of the simulation. The maximum value of the index is about 0.62 in Figure 6(a) and 6(d). These values indicate that there is a considerable symbiotic relationship for all the factory agents in the IES. 
(a)
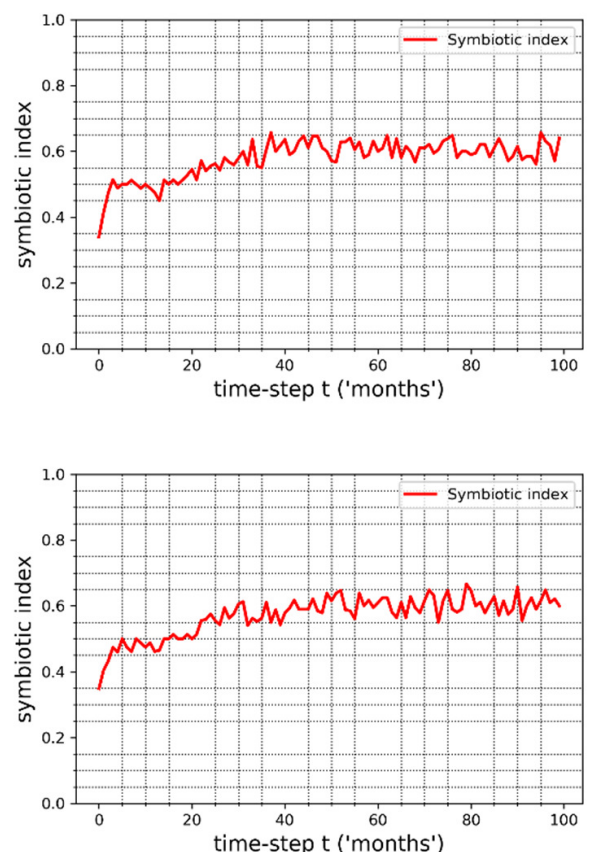

(c)

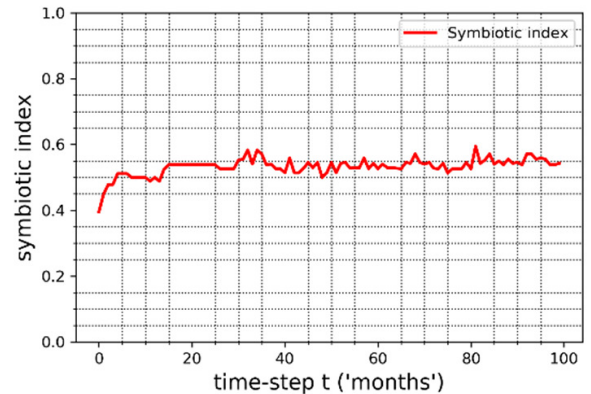

(d)

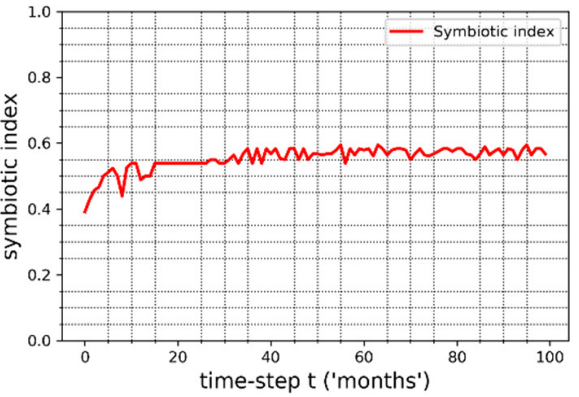

Fig. 6 SRI of the IES: (a) ST I, (b) ST II, (c) ST III, (d) ST IV.

\section{Sensitivity Analysis}

In order to gain some insight to the behavior of the factory agents in the IES, this section conducts the following sensitivity analyses regarding selling price increase on the SRI: (1) the effect of the increase by the factory agents and (2) the effect of the increase by market sellers. (a)
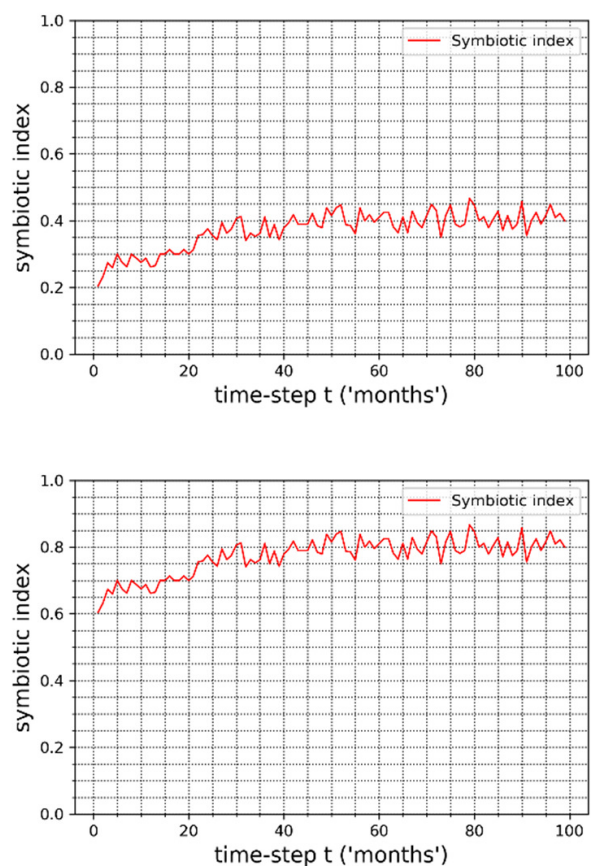

(c)

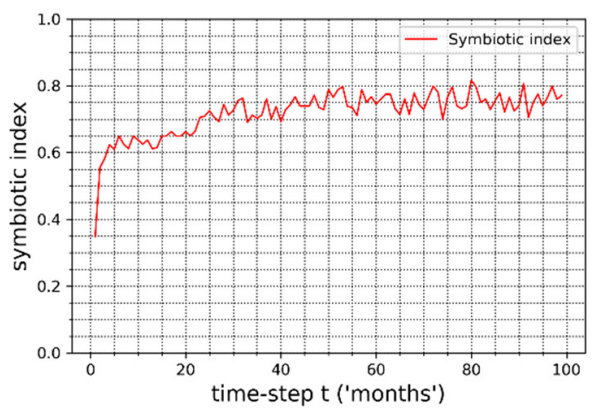

(d)

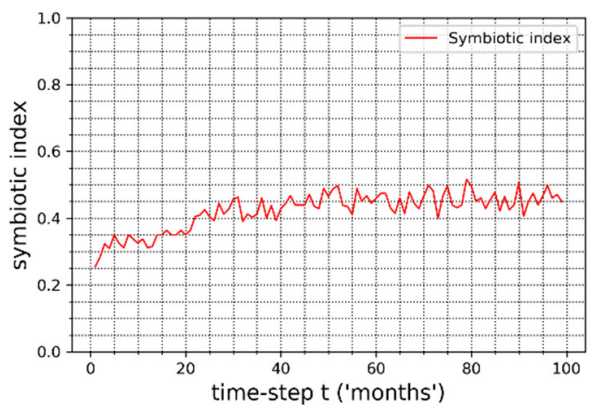

Fig. 7 Sensitivity analysis on the effect of high/low price: (a) SRI decreases between factory agents and buyers, (b) SRI increases between market sellers and buyers, (c) SRI increases between factory agents and buyers, and (d) SRI decreases between market sellers and buyers.

Sensitivity analysis is conducted to determine the effect of higher product prices from the factories in comparison to the market sellers. At the start of the simulation, the input values for the price of biogas, steam, and electricity were changed for only the factory agents and simulation was conducted using ST 
III. An increase in the range of $5 \%-10 \%$ was added to the initial input value, thus making the factory agent price higher than the market seller agents' while the other parameters remained the same. In Figures 7(a)-(b) it is seen that SRI decreases (average of 0.37) while it increases for the market sellers (average of 0.77 ), i.e. the buyers tend to form synergy with the market sellers than with the factory agents. In another sensitivity analysis, the market sellers had the high selling price at the start of the simulation while the other input parameters remained unchanged. Figures 7(c)-(d) show the impact of market sellers selling at higher price throughout the simulation run. The effects of this on the IES are that the factory agents' SRI to the buyers increases to 0.72 on average while the SRI for the market sellers to the buyers decreases to 0.40 . This behavior is similar to a self-sufficient system that does not require external source of supply but in reality this cannot happen because waste will always be generated. In summary, the results show that price variation has considerable impact on the configuration of the IES. It also suggests that in order to improve the existing relationships between the various parties in the IES, there must be some price regulation and this is one of the reasons why agents are able to learn from their history and the market's history.

\section{CONCLUSION}

As the sensitivity analysis carried out in this work showed, agent-based modeling technique is an effective tool that can be used to express the evolution of eco-industrial systems. With this modeling technique we can predict or simulate the price variation or forecast the demand and supply time series, which are difficult to be determined with supply and demand deterministic calculations. Increasing the product categories, extending industrial chains, and creating new industrial chains through utilizing wastes and by-products can create a comprehensive factory symbiotic community of higher sustainability. The results demonstrate that the Réseau agentbased model allowed the investigation of the different behaviors exhibited by the various agents in exchange of materials in an industrial park. This complex case study demonstrated realistic demand time series generated for decision strategies ST II and ST III, although the price curves for such strategies are not so realistic as they converge to a single value. In all, it was observed that the risk based seller decision strategy developed in this work provides significantly more realistic demand and supply time series, independent on whether the buyer chooses the seller randomly or according to the best price. This is the result of the risk-based price being linked to the market average only. In reality, it will also depend on manufacturing costs.

In conclusion, the findings of this work suggest that agentbased modeling is a promising tool that can be used to simulate industrial ecosystems in order to examine the behavior of agents in response to demand and supply variations.

\section{ACKNOWLEDGMENT}

This research is supported by the Tertiary Education Trust Fund (TETFUND, Nigeria) and the European Union's Horizon 2020 research and innovation program under SPIRE-06-2015
Energy and resource management systems for improved industry efficiency under the Grant Agreement no. 680843.

\section{REFERENCES}

[1] R. R. Heeres, W. J. V. Vermeulen, and B. de Walle, "Eco-industrial park initiatives in the USA and the Netherlands: first lessons," Journal of Cleaner Production, vol. 12, no. 8-10, pp. 985-995, Oct. 2004, doi: 10.1016/S0959-6526(04)00087-3.

[2] M. Mirata, "Experiences from early stages of a national industrial symbiosis programme in the UK: determinants and coordination challenges," Journal of Cleaner Production, vol. 12, no. 8-10, pp. 967983, Jan. 2004, doi: 10.1016/j.jclepro.2004.002.031.

[3] M. Mirata and R. Pearce, "Industrial Symbiosis in the UK," in Industrial Ecology and Spaces of Innovation, 2006, pp. 77-105.

[4] M. Mirata and T. Emtairah, "Industrial symbiosis networks and the contribution to Environmental Innovation: the Case of the Landskrona Industrial Symbiosis Programme," Journal of Cleaner Production, vol. 13, no. 10-11, pp. 993-1002, Aug. 2005, doi: 10.1016/j.jclepro.2004.12.010.

[5] B. R. Allenby and D. J. Richards, Eds., The Greening of Industrial Ecosystems. Washington, DC: National Academy of Engineering, 1994.

[6] N. Jacobsen, "Industrial Symbiosis in Kalundborg, Denmark: A Quantitative Assessment of Economic and Environmental Aspects," Journal of Industrial Ecology, vol. 10, no. 1-2, pp. 239-255, Jan. 2006, doi: 10.1162/108819806775545411.

[7] L. Fraccascia, V. Albino, and A. Garavelli, "Technical efficiency measures of industrial symbiosis networks using enterprise input-output analysis," International Journal of Production Economics, vol. 183, pp. 273-286, Jan. 2017, doi: 10.1016/j.ijpe.2016.11.003.

[8] V. Grimm et al., "A standard protocol for describing individual-based and agent-based models," Ecological Modelling, vol. 198, pp. 115-126, Jan. 2006.

[9] M. Martin, J. Ivner, N. Svensson, and M. Eklund, "Classification of Industrial Symbiosis Synergies: Application in the Biofuels Industry," presented at the Industrial Symbiosis and Eco-Industrial Parks, Aalborg, Denmark, Jun. 2009.

[10] V. Gonela, Stochastic Optimization of Sustainable Industrial Symbiosis Based Hybrid Generation Bioethanol Supply Chains. North Dakota: North Dakota State University, 2013.

[11] V. Gonela and J. Zhang, "Design of the optimal industrial symbiosis system to improve bioethanol production," Journal of Cleaner Production, vol. 64, pp. 513-534, Feb. 2014, doi: 10.1016/j.jclepro.2013.07.059.

[12] M. T. M. Espino and L. M. Bellotindos, "A System Dynamics Modeling and Computer-based Simulation in Forecasting Long-term Sufficiency: A Philippine Chicken Meat Sector Case Study," Engineering, Technology \& Applied Science Research, vol. 10, no. 2, pp. 5406-5411, Apr. 2020.

[13] N. Bichraoui, B. Guillaume, and A. Halog, "Agent-based Modelling Simulation for the Development of an Industrial Symbiosis - Preliminary Results," Procedia Environmental Sciences, vol. 17, pp. 195-204, Dec. 2013, doi: 10.1016/j.proenv.2013.02.029.

[14] D. Batten, "Fostering Industrial Symbiosis With Agent-Based Simulation and Participatory Modeling," Journal of Industrial Ecology, vol. 13, no. 2, pp. 197-213, Apr. 2009, doi: 10.1111/j.15309290.2009.00115.x.

[15] K. Cao, X. Feng, and H. Wan, "Applying agent-based modeling to the evolution of eco-industrial systems," Ecological Economics, vol. 68, no. 11, pp. 2868-2876, Sep. 2009, doi: 10.1016/j.ecolecon.2009.06.009.

[16] M. Chertow and J. Ehrenfeld, "Organizing Self-Organizing Systems," Journal of Industrial Ecology, vol. 16, no. 1, pp. 13-27, Feb. 2012, doi: 10.1111/j.1530-9290.2011.00450.x.

[17] D. Behrens, T. Schoormann, and R. Knackstedt, "Developing an Algorithm to Consider Mutliple Demand Response Objectives," Engineering, Technology and Applied Science Research, vol. 8, no. 1, pp. 2621-2626, Feb. 2018. 
[18] J. Raimbault et al., "A spatial agent based model for simulating and optimizing networked eco-industrial systems," Resources, Conservation and Recycling, vol. 155, Apr. 2020, doi: 10.1016/j.resconrec.2019.104538, Art no. 104538.

[19] T. Duong and T. Nguyen, "Network Reconfiguration for an Electric Distribution System with Distributed Generators based on Symbiotic Organisms Search," Engineering, Technology and Applied Science Research, vol. 9, no. 6, pp. 4925-4932, Nov. 2019.

[20] J. D. Farmer and D. Foley, "The economy needs agent-based modelling," Nature, vol. 460, pp. 685-686, Aug. 2009, doi: 10.1038/460685a.

[21] A. Borshchev and A. Filippov, "From System Dynamics and Discrete Event to Practical Agent Based Modeling: Reasons, Techniques, Tools," presented at the 22nd International Conference of the System Dynamics Society, Oxford, England, Jul. 2004.

[22] M. R. Ghali, J.-M. Frayret, and C. Ahabchane, "Agent-based model of self-organized industrial symbiosis," Journal of Cleaner Production, vol. 161, pp. 452-465, Sep. 2017, doi: 10.1016/j.jclepro.2017.05.128.

[23] G. O. Ajisegiri and F. L. Muller, "Effect of price dynamics in the design of eco-industrial parks: An agent-based modelling approach," presented at the 8th International Conference on Simulation and Modeling Methodologies, Technologies and Applications, Porto, Portugal, Jul. 2018, pp. 83-90.

[24] D. M. Yazan, V. A. Romano, and V. Albino, "The design of industrial symbiosis: an input-output approach," Journal of Cleaner Production, vol. 129, pp. 537-547, Aug. 2016, doi: 10.1016/j.jclepro.2016.03.160.

[25] B. P. Zeigler, T. G. Kim, and H. Praehofer, Theory of modeling and simulation. Cambridge, USA: Academic Press, 2000.

[26] K. M. Carley, Validating Computational Models. Pittsburgh, Pennsylvania: Carnegie Mellon University, 1996.

[27] D. Chassin, S. Behboodi, C. Crawford, and N. Diilali, "Agent-Based Simulation for Interconnection-Scale Renewable Integration and Demand Response Studies," Engineering, vol. 1, no. 4, pp. 422-435, Dec. 2015, doi: 10.15302/J-ENG-2015109.

[28] F. Klugl, "A validation methodology for agent-based simulations," presented at the ACM Symposium on Applied Computing, Fortaleza, Ceara, Brazil, Mar. 2008, pp. 39-43.

[29] G. O. Ajisegiri, "Application of an Agent Based Model to Study the Resource Exchanges within Eco-industrial Parks," Ph.D. dissertation, University of Leeds, Leeds, UK, 2019.

[30] V. Grimm, U. Berger, D. Deangelis, J. Polhill, J. Giske, and S. Railsback, "The ODD protocol: A review and first update," Ecological Modelling, vol. 221, no. 23, pp. 2760-2768, Nov. 2010, doi: 10.1016/j.ecolmodel.2010.08.019.

[31] V. Grimm et al., "Pattern-Oriented Modeling of Agent-Based Complex Systems: Lessons from Ecology," Science, vol. 310, no. 5750, pp. 987991, Dec. 2005, doi: 10.1126/science.1116681.

[32] L. Appels et al., "Anaerobic Digestion in Global Bio-Energy Production: Potential and Research Challenges," Renewable and Sustainable Energy Reviews, vol. 15, no. 9, pp. 4295-4301, Dec. 2011, doi: 10.1016/j.rser.2011.07.121. 